\title{
Front-surface fabrication of moderate aspect ratio micro-channels in fused silica by single picosecond Gaussian-Bessel laser pulse
}

\author{
Xin Liu $^{1,2,3} \cdot$ Nicolas Sanner $^{3} \cdot$ Marc Sentis $^{3} \cdot$ Razvan Stoian $^{4} \cdot$ Wei Zhao $^{1} \cdot$ Guanghua Cheng $^{1} \cdot$ Olivier Utéza $^{3}$
}

Received: 27 October 2017 / Accepted: 29 January 2018 / Published online: 3 February 2018

(c) The Author(s) 2018. This article is an open access publication

\begin{abstract}
Single-shot Gaussian-Bessel laser beams of 1 ps pulse duration and of $\sim 0.9 \mu \mathrm{m}$ core size and $\sim 60 \mu \mathrm{m}$ depth of focus are used for drilling micro-channels on front side of fused silica in ambient condition. Channels ablated at different pulse energies are fully characterized by AFM and post-processing polishing procedures. We identify experimental energy conditions (typically $1.5 \mu \mathrm{J}$ ) suitable to fabricate non-tapered channels with mean diameter of $\sim 1.2 \mu \mathrm{m}$ and length of $\sim 40 \mu \mathrm{m}$ while maintaining an utmost quality of the front opening of the channels. In addition, by further applying accurate post-polishing procedure, channels with high surface quality and moderate aspect ratio down to a few units are accessible, which would find interest in the surface micro-structuring of materials, with perspective of further scalability to meta-material specifications.
\end{abstract}

\section{Introduction}

Short laser pulses of (sub)-ps range find great interests in material processing, notably in fields like micro-machining for surface functionalization during last few decades [1-3]. Under intense irradiation of short pulse, dielectrics first experience moderate excitation via nonlinear photoionization; then, the generated conduction band electrons undergo rapid multiplication by repeated process of laser heating (inverse Bremsstrahlung) and impact ionization. As the free electron density goes above a critical value, plasma gradually turns highly absorbing yielding to optical breakdown and further to material ablation when the deposited energy is sufficiently high [4]. In surface ablation regime, for Gaussian beam with pulse duration especially below $100 \mathrm{fs,}$ a plasma shielding effect (solid density plasma mirror) may take place transiently, which contributes to self-limiting the

Xin Liu

liu@lp3.univ-mrs.fr

1 State Key Laboratory of Transient Optics and Photonics, Xi'an Institute of Optics and Precision Mechanics of CAS, Xi' an 710119, China

2 University of Chinese Academy of Sciences, Beijing 100049, China

3 Aix-Marseille University, CNRS, LP3 UMR 7341, 13288 Marseille, France

4 Laboratoire Hubert Curien, UMR CNRS 5516, Université de Lyon, Université Jean Monnet, 42000 Saint Etienne, France ablation depth to hundreds of nanometers at most [5]. This offers excellent control of making modest-depth craters with high regularity in the front opening. For large dimension processing purposes like deep drilling, longer pulses of picosecond (ps) duration are considered, since they offer increased penetration depth. However, Gaussian ps pulses yield morphological qualities like taper-profile inducing unwanted channel distortions. Moreover, independent manipulation of diameter $\varnothing$ and depth of channels $L$ on a very broad range of aspect ratio $(L / \varnothing)$ desirable for a flexible process are challenging with Gaussian beams. Therefore, a rather novel class of optical beams will be exploited here with the primary aim of precise fabrication.

Bessel beam (zero order of the first kind) appeared recently as a good solution in fabricating high aspect ratio channels due to its non-diffractive nature. In practice, a conical lens or axicon is usually used for generating the so-called Gaussian-Bessel beam which possesses finite energy and limited diffraction-free range, as a good approximation to ideal Bessel beam. The central core size of the beam can be determined by the half conical angle between the wave vector and the propagation axis. Due to the fact that the energy flux comes from the edge, a Bessel beam is claimed to be more resistant to nonlinear distortions during propagation compared to Gaussian beam. The plasma created on the axis at the front of the Bessel region should have less significant shielding and diffracting/defocusing effects on the incoming conical beam, and therefore, laser-matter interaction in farther regions is preserved. This property of Bessel beam 
is referred as 'self-healing' in another perspective $[6,7]$. Indeed, long cylindrical micro-channels with high aspect ratios $[8,9]$ up to 1200 have been demonstrated with singleshot ultrafast pulsed Bessel beams. The previous study with Bessel beam of variable pulse duration [10] also suggested that ps pulses are more beneficial for high efficiency energy deposition and could avoid temporal dynamics effects [11, 12] which can compromise the stationarity of the Bessel beam propagation. Bhuyan et al. [13] demonstrated that rear-side ablation under water immersion is a good strategy to facilitate debris evacuation and for obtaining taper-free micro/nano channel fabrication. However, rear-side ablation presents intrinsic limitations: (1) it is hardly applicable to multilayer samples, due to uncontrolled beam propagation in the thickness preceding the layer of interest, or even inapplicable to samples with non-transparent interlayer and (2) it still faces the limitation of the thickness of samples imposed by the working distance of the focusing objective.

In view of above issues, we investigate the fabrication of micro-channels on the front surface of fused silica sample under ambient condition. Temporally stretched (1 ps) single-shot Gaussian-Bessel beam with $\sim 0.9 \mu \mathrm{m}$ central core diameter and $\sim 60 \mu \mathrm{m}$ depth of focus (DOF) at full-width half-maximum (FWHM) is used in this paper (see Sect. 2). Single shot is justified because of the importance of achieving high throughput for drilling applications. In Sect. 3, we further vary the incident energy to define optimized conditions for high-quality drilling of micro-channels of moderate aspect ratio. The morphology of the machined channels is revealed by AFM measurements and confocal microscopy associated with post-processing polishing procedures. They are analyzed with respect to the quality of the front opening of craters, the accessible depth, and the uniformity of the outcome. Our strategy allows us to identify experimental conditions suitable to fabricate micro-channels of moderate length and high uniformity while maintaining high quality of the front opening of the channels. By further combining with post-polishing, we show that flexible aspect ratio down to a few units can be accessible.

\section{Experimental setup}

The experiment is performed with the beam line $5 \mathrm{~A}$ $(1 \mathrm{~mJ}, 100 \mathrm{~Hz}, 25 \mathrm{fs}$, linearly polarized, $800 \mathrm{~nm}$ ) of the Ti:Sapphire ASUR laser platform (Applications des Sources Ultra-Rapides, http://www.lp3.univ-mrs.fr) of LP3 laboratory. The initial laser beam diameter of $8 \mathrm{~mm}$ (measured at $1 / e^{2}$ of intensity) is reduced to $5 \mathrm{~mm}$ by introducing a two-spherical-lens telescopic system (not shown here) in view of two considerations: (1) inserting a pinhole at the confocal plane helps to filter out an uniform Gaussian beam and (2) reducing the size of the input beam favors the reduction of the final DOF of the micro-Bessel beam, which fits our aim of fabricating moderate aspect ratio channels. The glass sample located in the micro-Bessel zone is mounted on a sample holder with capability of precise $x, y, z$ translation and 3D angular adjustment. Single-pulse mode operation is available from a synchronized mechanical shutter. Pulse energy can be varied by a motorized half-wave plate in combination with a Brewster polarizer. All electromechanical devices are computer controlled via a Labview program.

To generate the micro-Bessel beam, we use a small base angle $\left(1^{\circ}\right)$ axicon (Altechna, 1-APX-2-H254-P, $n=1.45$ ) which provides the first Bessel region just next to it, as shown in Fig. 1a. Then, a 4-f system, made of a plane convex lens $\left(f_{l}=500 \mathrm{~mm}\right)$ and an objective lens $(20 \times, N A=0.4$, $f_{2}=10 \mathrm{~mm}$, Mitutoyo NIR, working distance $20 \mathrm{~mm}$ ) and showing a demagnifying factor of $\gamma=f_{1} / f_{2}=50$, is inserted to re-image the first Bessel region to the final Bessel region to increase the half conical angle $\theta$ of the Gaussian-Bessel beam from $0.45^{\circ}$ to $21.4^{\circ}$ and to reduce the DOF from $318.2 \mathrm{~mm}$ to $127.3 \mu \mathrm{m}$ (in the air) according to the equation $D O F=w / \tan \theta$, where $w$ is the radius size of incident beam. The FWHM diameter size of the central core of the beam can be theoretically approximated as $d \approx 2.4 /(k \sin \theta)=0.84$ $\mu \mathrm{m}$, where $k$ is the wave vector in vacuum. The final Bessel zone is collinearly imaged through a $50 \times$ objective $(\mathrm{NA}=0.55)$ lens and a tube lens $(f=200 \mathrm{~mm})$ on a beam analyzer (Gentec beamage, with pixel size of $6.45 \mu \mathrm{m}$ ). By scanning 150 different sections continuously along the optical axis with $1 \mu \mathrm{m}$ step and stacking them together, the 3D intensity distribution of the beam is constructed. Original and terminal positions are determined according to the location, where the intensity decays at $5 \%$ of the peak intensity of the central lobe. The longitudinal intensity profile can be thus extracted and recombined, as shown in Fig. 1b. A slight tapering shape of the central core is observed (also visible in Fig. 1d). We attribute it to the imperfection of the axicon, since a blunt apex of the axicon would produce some leaky light with low spatial frequencies, which contribute to the trumpet-shaped distribution of the beam at the beginning of the Bessel zone. Details about this aspect can be found in Ref [14]. The initial quality of the incident Gaussian beam could also be incriminated (intensity and spatial phase distribution), but in our case, a Gaussian beam with far-field pinhole filtering did not improve the tapering feature of the Bessel beam. The mean core size of the beam at FWHM is estimated to be $\sim 0.9 \mu \mathrm{m}$ according to the measurement. Axial peak intensities are plotted in Fig. 1c, and FWHM of DOF is estimated to be $60 \mu \mathrm{m}$. Four section images of the beam at the location of $Z=20,40,60$, and $80 \mu \mathrm{m}$ are demonstrated in Fig. 1d. Each image is normalized to the maximum intensity to better visualize the beam spot. 


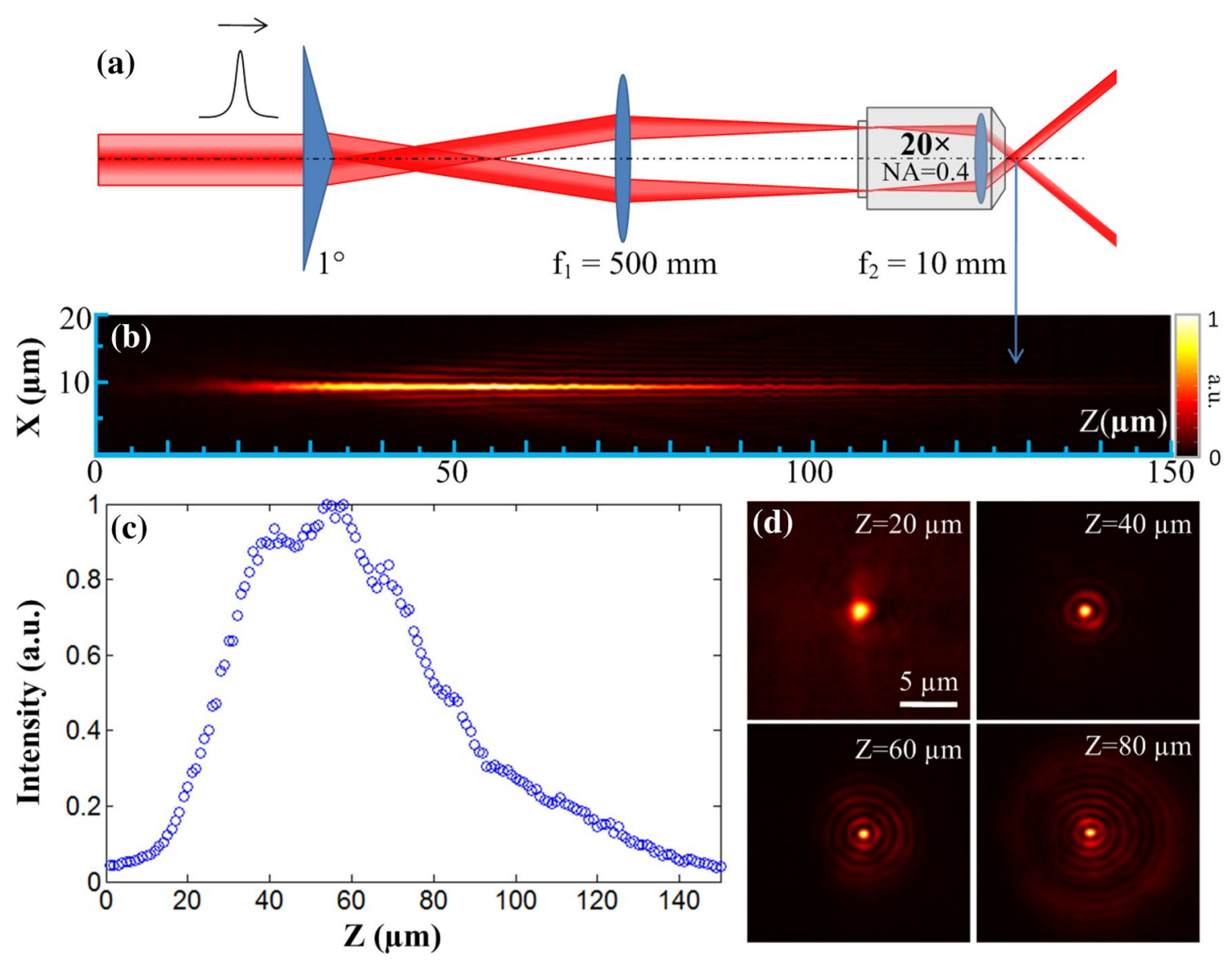

Fig. 1 Characterization of the Gaussian-Bessel beam in the air. a Schematic diagram of 4-f setup for generating the desired beam. b Reconstructed beam intensity profile along the optical axis. $\mathbf{c}$ Beam

\section{Results and discussion}

The generated micro-Bessel beam is used to fabricate channels on the front surface of glass sample ( $4 \mathrm{~mm}$-thick fused silica, Corning 7980-5F) under ambient condition in single-shot regime. The adjacent channels are separated by 20 $\mu \mathrm{m}$ to prevent potential mutual influence. Pulse energy is measured before the target sample surface and varied from 0.6 to $6 \mu \mathrm{J}$ to determine the optimized conditions for channel fabrication. The laser pulses are negatively chirped to 1 ps by tuning the grating position in the laser compressor. At high energy, the plasma channel triggered in the air by the micro-Bessel beam explicitly indicates, where the beam is spatially located. It is imaged from the side by a $10 \times$ objective lens and a CCD camera, which is helpful for precisely positioning the sample. The front surface of the sample is positioned around the peak intensity point of the line focus for all cases (around $Z=50 \mu \mathrm{m}$, as shown in Fig. 1b). Note that high incident angle of $\theta_{\text {air }}=21.4^{\circ}$ is applied, the refracted beam inside fused silica $(n=1.45)$ has an angle of $\theta_{\text {glass }}=14.6^{\circ}$, and thus, the line focus inside peak intensity along the optical axis. d Selected transverse section images of the beam; the spatial scale bar is applicable for all four images

the glass sample will stretch along the longitudinal axis by a factor of $\tan \theta_{\text {air }} / \tan \theta_{\text {glass }} \approx 1.51$ in linear propagation regime. After irradiation, modification conditions in bulk are revealed by $50 \times$ transmission optical microscopy and depicted as a function of pulse energy, as shown in Fig. 2. The dark laser-modified elongated regions are confirmed to be voids in the following by the polishing procedure. Thanks to the long picosecond pulse, undesired plasma effects related to high free electron density largely exceeding the critical density (i.e. defocusing) are sufficiently minimized before energy is deposited to the target zone. Therefore, the length of channels can be adjusted according to the applied pulse energy. At high energy $(E>3 \mu \mathrm{J})$, side effects related to the lobe rings of Bessel beam are visible in bulk around the core region. Moreover, some irregular modulations are observed along the channels, which confirm that working at high energy are non-favorable processing conditions. As energy is dropped from 3 to $1.5 \mu \mathrm{J}$, the channels gradually appear with shorter length and better uniformity. The insets on the top of the channels are the corresponding surface morphologies (from 3 to $1 \mu \mathrm{J}$ ) characterized by atomic force 


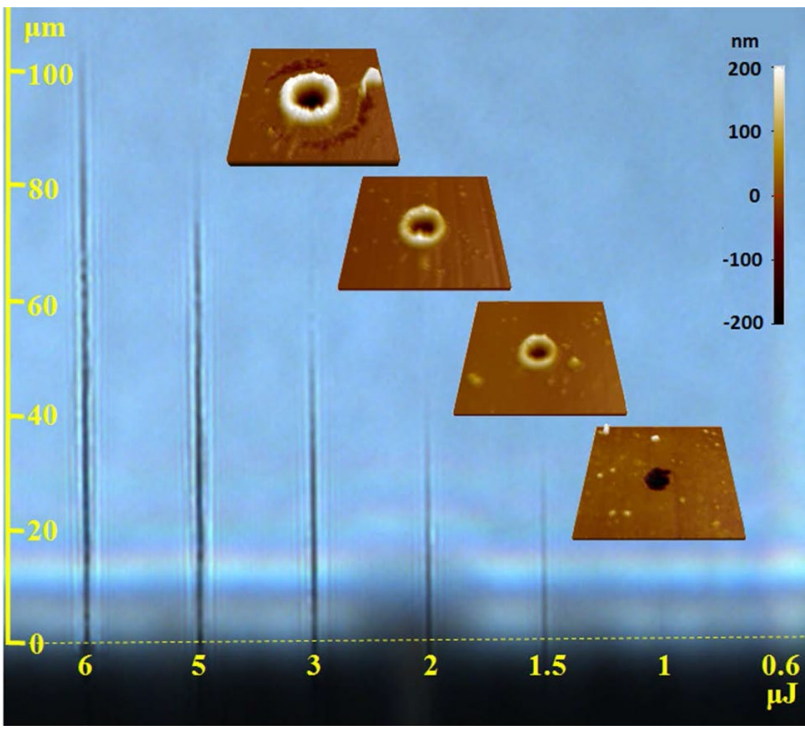

Fig. 2 Transmission optical microscope reveals the evolution of bulk modification as a function of the pulse energy (from 6 to $0.6 \mu \mathrm{J}$ ). Laser beam comes from the bottom of the image. The insets $(5 \times 5$ $\mu \mathrm{m}^{2}$ ) on the top of the channels are the corresponding surface morphologies (from 3 to $1 \mu \mathrm{J}$ ) characterized by atomic force microscopy (AFM, PSIA XE-100). Scale bar describing the vertical axis is added for reference

microscopy (AFM, PSIA XE-100). For $1 \mu \mathrm{J}$ case, a crater with an opening of $\sim 0.9 \mu \mathrm{m}$ is obtained with neither rims on the edge nor circular side rings arising from Bessel beam intensity distribution. This energy operating condition corresponds to an interesting processing condition to obtain a high-quality front-opening channel, without the need of any post-processing. No surface modification is observed with the energy of $0.6 \mu \mathrm{J}$.

Polishing procedures are carried out from the side, to directly reveal the longitudinal section of channels. Since the diameters of channels are very small ( $1 \mu \mathrm{m}$ range), it is quite challenging to reach a single channel right in the center. To address this problem, channels are closely arranged as a spot matrix (10 $\mu \mathrm{m}$ separation), and the rows of the matrix are oriented at an angle of $1.5^{\circ}$ with respect to sample edge to ensure that we reach at least one channel cross section when applying the polishing procedure (see the schematic diagram in Fig. 3a). Figure 3b shows a longitudinal section of channel measured by AFM ( $3 \mu \mathrm{J}$ case). The $\sim 36 \mu \mathrm{m}$-long trench explicitly confirms the void nature, the continuity, and the good regularity of the channel. However, to be sure that we measure the whole channel, it is also required that the channels axes are strictly parallel to the polishing disk. A possible small angle can occur when doing the polishing, which leads to only partial exposure of the channels. This partly justifies the difference between the length measured by this procedure and the length of the dark region in Fig. 2. Thus, although AFM enables quantitative measurements of

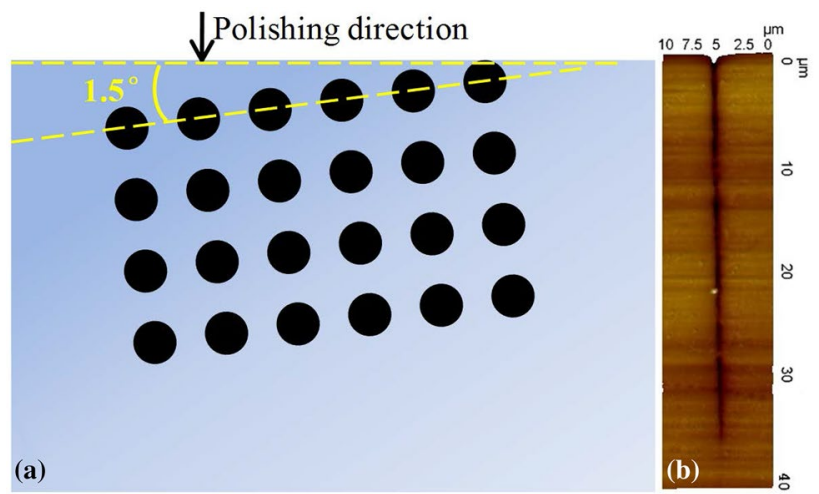

Fig. 3 a Scheme of principle for successful application of post-polishing procedure: an array of channels is fabricated to enhance the hitting possibility to expose the axial profile of channels during the polishing procedure. $\mathbf{b}$ Partially exposed longitudinal section of channel ( $3 \mu \mathrm{J}$ case $)$ is characterized by AFM

the channel diameter all along its section, this limitation prevents us to use this technique to safely assess the cylindricity of the channel.

To get a quantitative characterization of the channels, another complementary technique is developed. The orthogonal surfaces are gradually polished layer by layer to reveal the transverse sections of channels. Following the specifications of our auto-polishing machine (PRESI Company), a special lint disk combined with the $2.5 \mu \mathrm{m}$ cerium oxide suspension is used for the final polishing of glass sample. The parameters of $1.5 \mathrm{daN}$ force, $300 \mathrm{RPM}$, and $30 \mathrm{~s}$ time duration are applied for each polishing step, which yield a typical average removal thickness of $6.7 \mu \mathrm{m}$ (since in total $80 \mu \mathrm{m}$ was removed in 12 steps). Even if the polishing powder used here has a size comparable to the diameter of the laser-processed channels, the quality of the surface after polishing is excellent, exhibiting optical quality with average roughness less than $30 \mathrm{~nm}$ according to AFM measurement. Note, however, that we cannot completely work out the eventuality that the opening of the channel on each layer is systematically enlarged by the grinding effect related to the applied polishing pressure. The morphology of the layers is analyzed by confocal microscopy (Leica DCM 3D) after each polishing cycle thus enabling us to reconstruct the profile of the channels. To precisely measure the material thickness at each step after polishing, specific marks are laser-inscribed on the side wall of the sample perpendicular to the surface on which the micro-channels have been drilled. Therefore, we clearly know how much thickness of the sample is removed, and the depth coordinate of the layers can be defined. Figure 4 shows the tomography of channels including the visualization of the first five layers and of the last layer in three cases $(6,3$, and $1.5 \mu \mathrm{J})$. At high energy of $6 \mu \mathrm{J}$, the imprint typical of Bessel beam pattern is seen at the surface and the footprint of the first side lobe can still be 

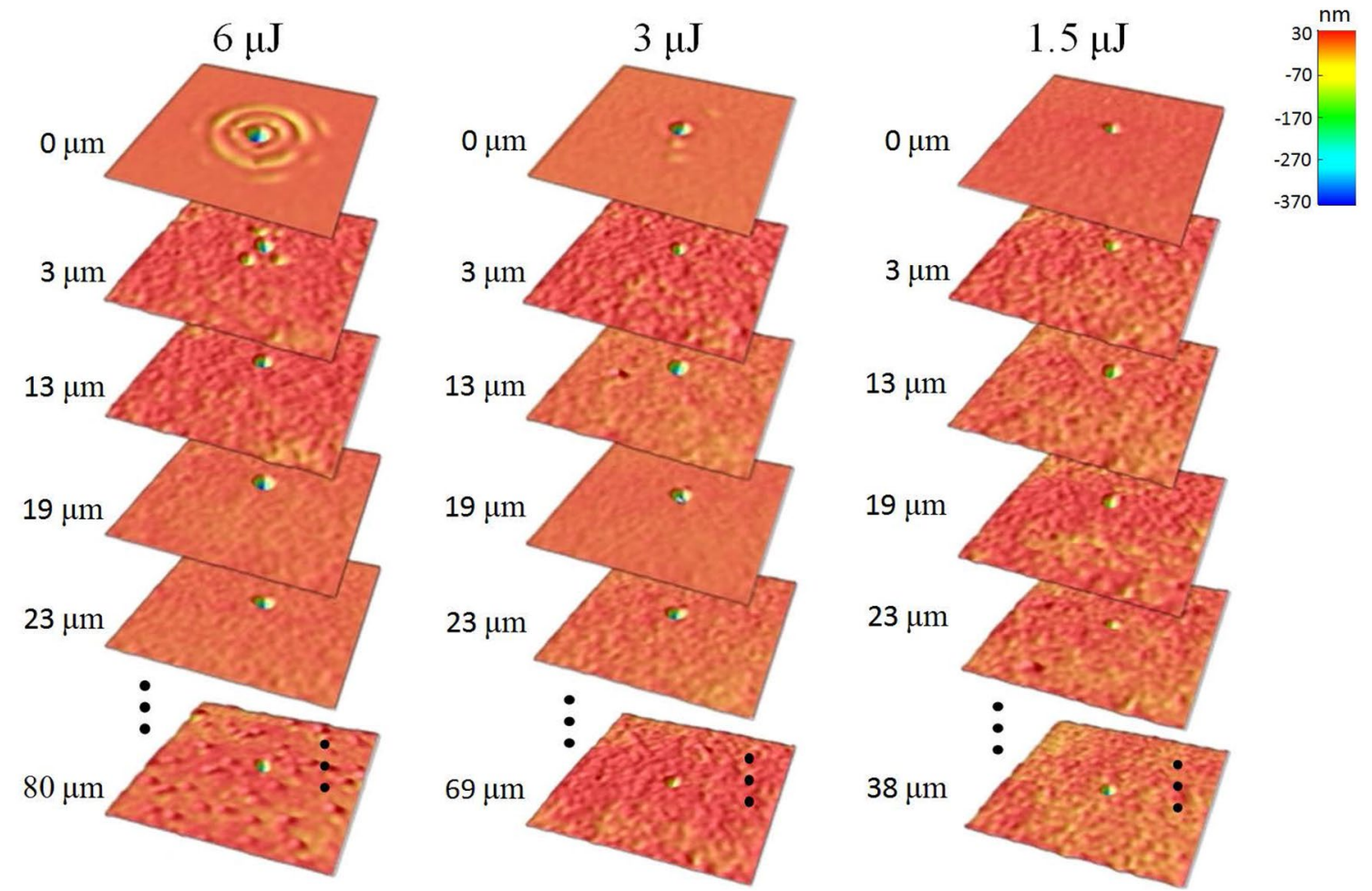

Fig. 4 Tomography of channels is revealed by layer-by-layer polishing and surface imaging with confocal microscopy in 6,3 , and 1.5 $\mu \mathrm{J}$ cases. All layer images have the size of $14 \times 14 \mu \mathrm{m}^{2}$. The depth

resolved $3 \mu \mathrm{m}$ below the surface, which reinforce the previous comment that working at high energy do not promote favorable conditions for fabricating high-quality channels. Note that in the $6 \mu \mathrm{J}$ case, we terminate the polishing procedure at $\sim 80 \mu \mathrm{m}$ depth. Channels with clean surface and good cylindrical shape are obtained at low energy of 3 and $1.5 \mu \mathrm{J}$, and the last layer with recognizable modification locates at 69 and $38 \mu \mathrm{m}$, respectively. For each diameter measurement, due to the experimental uncertainty on the coordinate of the surface reference, we estimate the maximal and minimal values of channel diameter in orthogonal directions (totally four values) from confocal microscopy measurement. The average values of diameter with related uncertainty are presented with respect to layer positions for all energy cases, as shown in Fig. 5. The average diameter along the whole channel is calculated to be $1.53,1.37$, and $1.24 \mu \mathrm{m}$ for 6 , 3 , and $1.5 \mu \mathrm{J}$ accordingly. Channels in all cases generally demonstrate good cylindrical quality. Considering the three cases of energy mentioned, and the total length on which these channels are drilled, the maximum standard deviation of diameter is below $170 \mathrm{~nm}$, which is reasonable considering the reading error from the inherent $110 \mathrm{~nm}$ transverse sampling resolution of confocal microscopy. More accurate characterization and precise vertical removal rate could be achieved by applying low polishing pressure or using of each layer with respect to the surface is indicated on the left. A color scale is added on the up-right corner of the image to highlight the hole structure on the surface

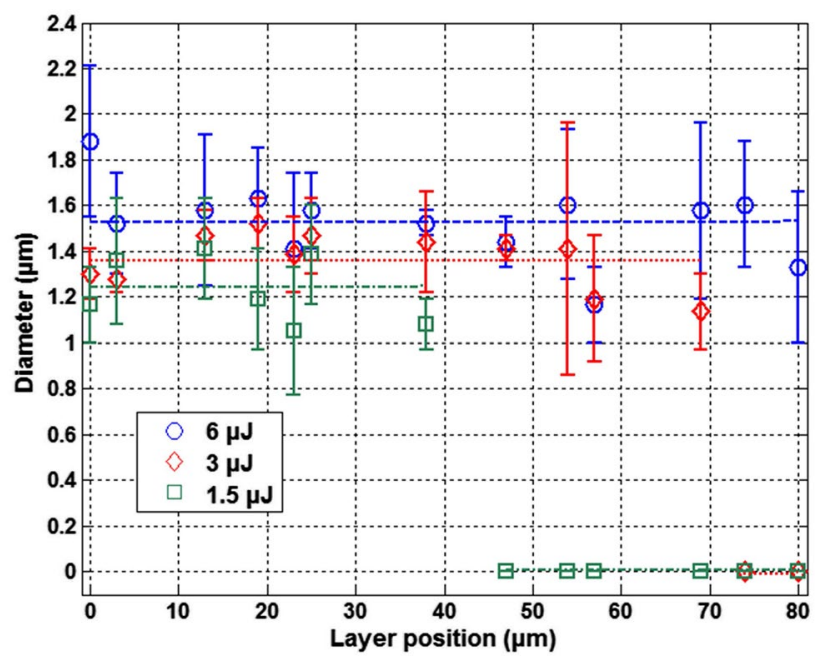

Fig. 5 Diameter of channel versus layer position and for different pulse energy cases $(6,3$, and $1.5 \mu \mathrm{J})$. Data are extracted from tomography measurement in Fig. 4 and the diameter is estimated as the average size of the orthogonal directions. The dashed lines are added to guide the eye

nano-sized polishing powder. Therefore, it is expected that laser ablation combined with post-polishing process can be a strategy to make channels with good surface quality and 
aspect ratio down to a few units. As an example, if we consider the case $1.5 \mu \mathrm{J}$, the measured diameter after polishing a thickness of $38 \mu \mathrm{m}$ is approximately $1.1 \mu \mathrm{m}$ and the remaining depth is at maximum $9 \mu \mathrm{m}$ (next polished layer showing no hole, see Fig. 5) corresponding to an aspect ratio close to 8 . This range of aspect ratio is well-suited for the fabrication of advanced optical components like photonic crystal structures, upon further downscaling $[15,16]$.

\section{Conclusion}

We demonstrate single-shot (important for high throughput) technical route to fabricate at the front surface of glass sample taper-free channels with good opening quality, micrometric diameter, and few tens of micrometer length. Optimized energy conditions are defined typically corresponding to the use of $\sim$ micro-Joule pulse energy for 1 ps pulse duration. In addition, by applying accurate post-polishing procedure, channels with high surface quality and aspect ratio down to a few units are accessible. These results open promising prospects for structuring material surface with moderate aspect ratio in the micrometric domain. With this approach, we envision that either using even higher conical angle Bessel beam or doubling the laser frequency, one could down-size the spatial characteristics of the channels. This could also be interesting in view of developing new fabrication routes at the spatial scale below $\mu \mathrm{m}$ which is suitable for photonic crystal application and meta-materials in general.

Acknowledgements X. Liu, G. Cheng, and W. Zhao gratefully acknowledge financial support from China Scholarship Council (CSC No. 201504910604) and the National Natural Science Foundation of China (No. 61378019 and No. 41427804), and X. Liu thank D. Grojo, R. Clady, L. Charmasson and Jean-Luc Bellemain for helpful discussions and technical assistance. R. Stoian gratefully acknowledges the support of the "Agence Nationale de la Recherche", France (Project Smart-Lasir 11BS09026). N. Sanner, O.Utéza, and M. Sentis gratefully acknowledge the financial support of European Community, Ministry of Research and High Education, Region Provence-Alpes-Côte d'Azur, Department of Bouches-du-Rhône, City of Marseille, CNRS, and AixMarseille University for funding the ASUR platform.

Open Access This article is distributed under the terms of the Creative Commons Attribution 4.0 International License (http://creativeco mmons.org/licenses/by/4.0/), which permits unrestricted use, distribution, and reproduction in any medium, provided you give appropriate credit to the original author(s) and the source, provide a link to the Creative Commons license, and indicate if changes were made.

\section{References}

1. K. Sugioka, Y. Cheng, Appl. Phys. Rev. 1, 41303 (2014)

2. K.C. Phillips, H.H. Gandhi, E. Mazur, S.K. Sundaram, Adv. Opt. Photonics. 7, 684 (2015)

3. S. Mishra, V. Yadava, Opt. Lasers Eng. 73, 89 (2015)

4. P. Balling, J. Schou, Reports Prog. Phys. 76, 36502 (2013)

5. O. Utéza, N. Sanner, B. Chimier, A. Brocas, N. Varkentina, M. Sentis, P. Lassonde, F. Légaré, J.C. Kieffer, Appl. Phys. A. 105, $131(2011)$

6. D. Faccio, E. Rubino, A. Lotti, A. Couairon, A. Dubietis, G. Tamoauskas, D.G. Papazoglou, S. Tzortzakis, Phys. Rev. A. 85, 1 (2012)

7. D. McGloin, K. Dholakia, Contemp. Phys. 46, 15 (2005)

8. S. Mitra, M. Chanal, R. Clady, A. Mouskeftaras, D. Grojo, Appl. Opt. 54, 7358 (2015)

9. M.K. Bhuyan, F. Courvoisier, P.A. Lacourt, M. Jacquot, R. Salut, L. Furfaro, J.M. Dudley, Appl. Phys. Lett. 97, 9 (2010)

10. V. Garzillo, V. Jukna, A. Couairon, R. Grigutis, P. Di Trapani, O. Jedrkiewicz, J. Appl. Phys. 120, 13102 (2016)

11. P.K. Velpula, M.K. Bhuyan, F. Courvoisier, H. Zhang, J.P. Colombier, R. Stoian, Laser Photonics Rev. 10, 230 (2016)

12. P. Polesana, M. Franco, A. Couairon, D. Faccio, P. Di Trapani, Phys. Rev. A. 77, 1 (2008)

13. M.K. Bhuyan, F. Courvoisier, P.-A. Lacourt, M. Jacquot, L. Furfaro, M.J. Withford, J.M. Dudley, Opt. Express. 18, 566 (2010)

14. J. Dudutis, P. Gečys, G. Račiukaitis, Opt. Express. 24, 28433 (2016)

15. B. Momeni, J. Huang, M. Soltani, M. Askari, S. Mohammadi, M. Rakhshandehroo, A. Adibi, Opt. Express. 14, 2413 (2006)

16. G.W. Burr, S. Diziain, M.-P. Bernal, Opt. Express. 16, 6302 (2008) 